\title{
Measurements of jets in ALICE
}

\author{
Christine Nattrass ${ }^{*}$ \\ University of Tennessee, Knoxville \\ E-mail: christine.nattrasseutk.edu
}

High energy collisions of heavy nuclei permit the study of nuclear matter at high temperatures and energy densities. Under these conditions the fundamental theory for strong interactions, QCD, predicts a phase transition to a plasma of quarks and gluons. This matter, called a Quark Gluon Plasma (QGP), has been studied experimentally for the last decade and has been observed to be a strongly interacting liquid with a low viscosity. High energy partons created early in the collision interact with the QGP and provide unique probes to study its micsoscopic properties. Studies of these partons through full jet reconstruction and high-momentum particles have demonstrated that they lose energy in the medium, showing that the QGP is a strongly interacting, dense medium. Studies of jet-hadron correlations and jet structure can help understand the energy loss mechanism. We present measurements of jet spectra, and jet-hadron correlations, and jet structure in $\mathrm{Pb}-\mathrm{Pb}$ collisions and discuss the constraints these studies place on partonic energy loss mechanisms in the medium.

EPS-HEP 2017, European Physical Society conference on High Energy Physics 5-12 July 2017

Venice, Italy

\footnotetext{
*for the ALICE Collaboration

†peaker.
} 


\section{Introduction}

A hot, dense liquid of quarks and gluons called a Quark Gluon Plasma (QGP) is formed in high energy nuclear collisions $[1,2,3]$. This matter can be studied using the products of hard parton scatterings early in the collision. High momentum partons fragment into collimated sprays of particles called jets. These high momentum partons lose energy through interactions with the medium, a process known as jet quenching, and measurements of the modifications of jets in heavy ion collisions can be used to study the microscopic properties of the QGP $[4,5]$. Partonic interactions with the medium can lead to gluon bremsstrahlung and collisional energy loss, which results in broadening of jets and softening of jet constituents.

ALICE has measured several observables in order to quantify partonic energy loss and characterize the nature of partonic interactions with the medium. Precision measurements of jet spectra provide quantitative constraints on partonic energy loss. Jet-hadron correlations allow precision measurements of the average properties of jets, and several ALICE measurements are sensitive to modifications of jet structure. ALICE [6] is a general purpose detector with precision tracking and calorimetry optimized for the high multiplicity environment of heavy ion collisions. Measurements of jets primarily use the Inner Tracking System (ITS) [6], the Time Projection Chamber (TPC) [7], and the Electromagnetic Calorimeter (EMCal) $[8,9]$. The TPC and ITS provide tracking with high efficiency and momentum resolution above $150 \mathrm{MeV} / c$. The EMCal is a lead/scintillator sampling calorimeter covering $100^{\circ}$ in azimuth and $|\eta|<0.7$ in pseudorapidity. ALICE measures charged track jets and full jets. Charged track jet energies are corrected up to the energy of primary charged particles and use information from the tracking detectors. Full jets are corrected up to the full jet energy at the particle level and combine information from the tracking detectors and the EMCal. Clusters above $300 \mathrm{MeV}$ and tracks above $150 \mathrm{MeV} / \mathrm{c}$ are used for jet reconstruction. The track momentum is subtracted from clusters which are matched to tracks for full jet measurements in order to avoid double counting. The anti- $k_{T}$ jet-finding algorithm [10] is used with a boost-invariant $p_{\mathrm{T}}$ recombination scheme [11], meaning that jet momentum $p_{\mathrm{T}, \mathrm{jet}}$ is the scalar sum of the constituent momenta.

\section{Results}

The measurements of the nuclear modification factor, $R_{\mathrm{AA}}$, of jets demonstrate substantial suppression relative to binary collision scaling of the jet spectra in $p+p$ collisions $[12,13,14]$. Some of the energy lost by the parton may remain inside the jet cone, so measurements of jet spectra alone are not sufficient to characterize partonic energy loss. Reaction plane dependent measurements of jet-hadron correlations can constrain the path length dependence of medium modifications of jets [15]. The high multiplicity in heavy ion collisions leads to combinatorial jets comprising only particles from the background and a large combinatorial background in jet-hadron correlations from the production of particles uncorrelated with the hard scattering. The reaction plane fit method enables precision subtraction of the combinatorial background since the average background can be determined with higher accuracy than the background in individual jets [16]. The contribution from combinatorial jets is suppressed by using calorimeter clusters with $E>3.0 \mathrm{GeV}$ and tracks with $p_{\mathrm{T}}>3.0 \mathrm{GeV} / c$. The uncertainties partially cancel out when calculating the ratio 
of the number of associated particles for jets in-plane to the number of associated particles out-ofplane, shown in figure 1 for $R=0.4$ anti- $k_{T}$ full jets reconstructed with reconstructed jet momenta $20<p_{\mathrm{T}}^{\text {reco }}<40 \mathrm{GeV} / c$ as a function of associated particle momenta. This ratio is consistent with unity within uncertainties, indicating little path length dependence in the medium modifications of jets. It has been proposed that jet-by-jet fluctuations in the partonic energy loss may dominate over path length for some observables, leading to little apparent path length dependence for modifications of jets [17]. The results in figure 1 are consistent with this interpretation, indicating that the impact of fluctuations may be larger than that of path length.
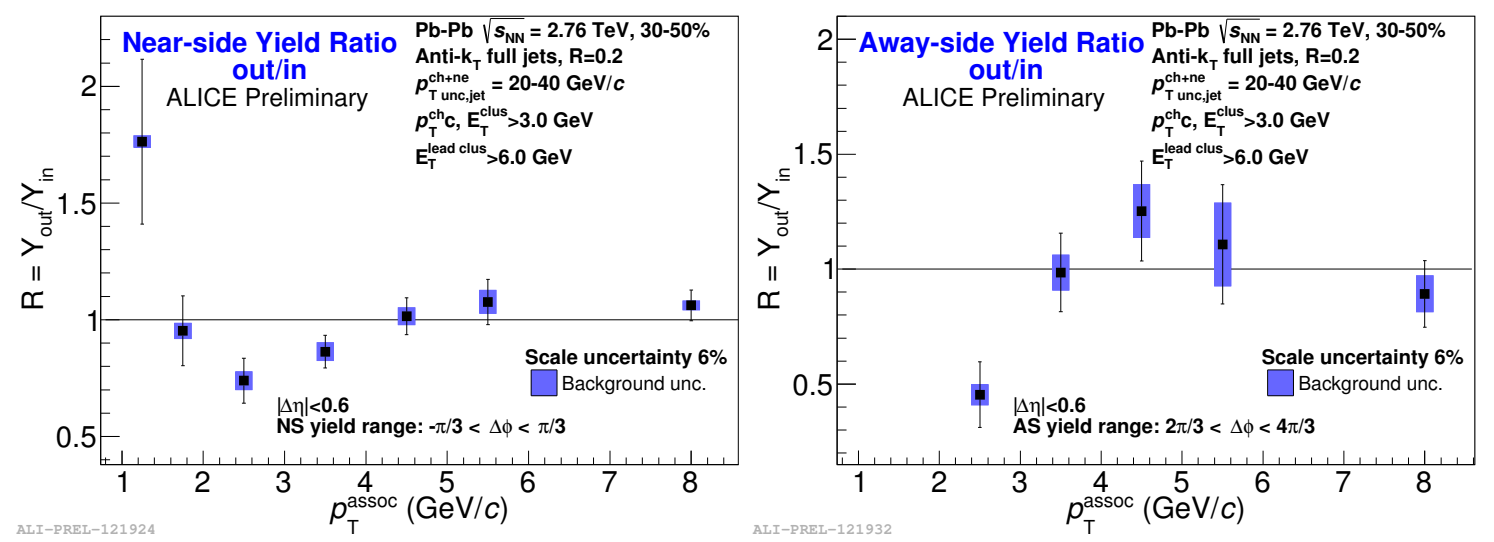

Figure 1: The ratio of the number of associated particles for jets in-plane to the number of associated particles out-of-plane for $R=0.4$ anti- $k_{T}$ full jets reconstructed with reconstructed jet momenta $20<p_{\mathrm{T}}^{\text {reco }}<40 \mathrm{GeV} / c$ as a function of associated particle momenta [15].
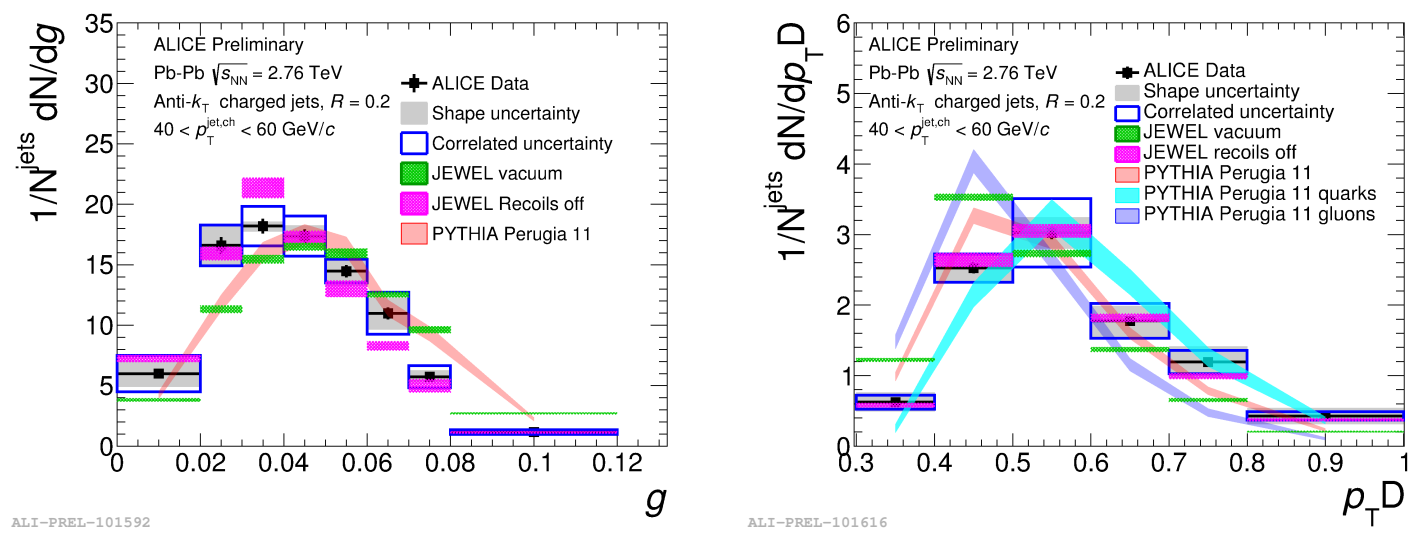

Figure 2: Measurements of the jet girth $g$ and the dispersion $p_{\mathrm{T}}^{D}$ for charged jets with $40<p_{\mathrm{T}}^{\text {ch }}<60 \mathrm{GeV} / c$. Measurements are compared to calculations using JEWEL [18] and the PYTHIA [19] Perugia 2011 tune [20].

ALICE has been actively investigating measurements which may provide greater discriminating power between models for interactions of jets in the medium. In the presence of a medium, the average jet mass may be higher than in vacuum [21] but measurements are consistent with no 
medium modification [22]. Measurements of modifications of jet structure in the presence of a medium may be able to distinguish between different models for the interaction of partons with the medium. The jet girth, $g$, is the $p_{\mathrm{T}}$ weighted width of the jet

$$
g=\sum_{i} \frac{p_{\mathrm{T}}^{i}}{p_{\mathrm{T}}^{\mathrm{jet}}}\left|r_{i}\right|,
$$

where $r_{i}$ is the angular distance between particle $i$ and the jet axis. If jets are broadened by the medium, $g$ would be higher in $\mathrm{Pb}+\mathrm{Pb}$ collisions than in $p+p$. Measurements of the girth shown in figure 2 indicate a slightly lower girth in $\mathrm{Pb}+\mathrm{Pb}$ collisions. The dispersion is given by

$$
p_{\mathrm{T}}^{D}=\sqrt{\Sigma_{i} p_{\mathrm{T}, i}^{2}} / \Sigma_{i} p_{\mathrm{T}, i}
$$

where $p_{T, i}$ is the momentum of the constituents. Measurements of $p_{\mathrm{T}}^{D}$ shown in figure 2 likewise indicate that jets in $\mathrm{Pb}+\mathrm{Pb}$ collisions are somewhat narrower than in $p+p$ collisions. It is unlikely that jets are collimated by interactions with the medium, but more likely that this indicates a bias towards unmodified jets or quark jets in $\mathrm{Pb}+\mathrm{Pb}$ collisions. Measurements are compared to calculations using the PYTHIA [19] Perugia 2011 tune [20] and JEWEL [18], a model incorporating partonic energy loss in the medium. JEWEL predicts more broadening than seen in the data. Measurements of LeSub, the difference between the leading and subleading particles' momenta, indicate that it is not modified through interactions with the medium [23].

\section{Conclusions}

Measurements of jet spectra in $\mathrm{Pb}+\mathrm{Pb}$ collisions indicate substantial medium modifications. These modifications do not result in a path length dependence in the number of constituents or modifications of the jet mass or LeSub. Measurements of the girth and the dispersion of jets surviving interactions with the medium indicate that jets are somewhat narrower than observed in $p+p$ collisions. These measurements indicate the importance of jet-by-jet fluctuations in partonic energy loss and survivor bias.

\section{References}

[1] ATLAS Collaboration, G. Aad et al., "Observation of a Centrality-Dependent Dijet Asymmetry in Lead-Lead Collisions at $\sqrt{s_{N N}}=2.77 \mathrm{TeV}$ with the ATLAS Detector at the LHC," Phys.Rev.Lett. 105 (2010) 252303, arXiv:1011.6182 [hep-ex].

[2] CMS Collaboration, S. Chatrchyan et al., "Study of high-pT charged particle suppression in $\mathrm{PbPb}$ compared to $p p$ collisions at $\sqrt{s_{N N}}=2.76 \mathrm{TeV}$," Eur.Phys.J. C72 (2012) 1945, arXiv:1202.2554 [nucl-ex].

[3] ALICE Collaboration, B. Abelev et al., "Measurement of Event Background Fluctuations for Charged Particle Jet Reconstruction in Pb-Pb collisions at $\sqrt{s_{N N}}=2.76 \mathrm{TeV}$," JHEP 1203 (2012) 053, arXiv:1201.2423 [hep-ex].

[4] M. Connors, C. Nattrass, R. Reed, and S. Salur, "Review of Jet Measurements in Heavy Ion Collisions," arXiv:1705.01974 [nucl-ex]. 
[5] L. Cunqueiro, "Jet Production at RHIC and LHC," in 26th International Conference on Ultrarelativistic Nucleus-Nucleus Collisions (Quark Matter 2017) Chicago,Illinois, USA, February 6-11, 2017.

[6] ALICE Collaboration, K. Aamodt et al., "The ALICE experiment at the CERN LHC," JINST 3 (2008) S08002.

[7] J. Alme et al., "The ALICE TPC, a large 3-dimensional tracking device with fast readout for ultra-high multiplicity events," Nucl. Instrum. Meth. A622 (2010) 316-367, arXiv: 1001.1950 [physics.ins-det].

[8] ALICE EMCal Collaboration, J. Allen et al., "Performance of prototypes for the ALICE electromagnetic calorimeter," Nucl. Instrum. Meth. A615 (2010) 6-13, arXiv:0912. 2005 [physics.ins-det].

[9] ALICE Collaboration, P. Cortese et al., "ALICE Electromagnetic Calorimeter Technical Design Report,” Tech. Rep. CERN-LHCC-2008-014. ALICE-TDR-14, CERN, Geneva, Aug, 2008.

[10] M. Cacciari, G. P. Salam, and G. Soyez, “The Anti-k(t) jet clustering algorithm,” JHEP 04 (2008) 063, arXiv:0802.1189 [hep-ph].

[11] M. Cacciari, G. P. Salam, and G. Soyez, "FastJet User Manual," Eur.Phys.J. C72 (2012) 1896, arXiv:1111.6097 [hep-ph].

[12] ALICE Collaboration, J. Adam et al., "Measurement of jet suppression in central $\mathrm{Pb}-\mathrm{Pb}$ collisions at $\sqrt{s_{\mathrm{NN}}}=2.76 \mathrm{TeV}, "$ Phys. Lett. B746 (2015) 1-14, arXiv:1502.01689 [nucl-ex] .

[13] ATLAS Collaboration, G. Aad et al., "Measurements of the Nuclear Modification Factor for Jets in $\mathrm{Pb}+\mathrm{Pb}$ Collisions at $\sqrt{s_{\mathrm{NN}}}=2.76 \mathrm{TeV}$ with the ATLAS Detector," Phys. Rev. Lett. 114 no. 7, (2015) 072302, arXiv:1411.2357 [hep-ex].

[14] CMS Collaboration, V. Khachatryan et al., "Measurement of inclusive jet cross-sections in pp and $\mathrm{PbPb}$ collisions at $\sqrt{s_{N N}}=2.76 \mathrm{TeV}$, arXiv:1609.05383 [nucl-ex] .

[15] ALICE Collaboration, J. Mazer, "Jet-hadron correlations relative to the event plane at the LHC with ALICE,” J. Phys. Conf. Ser. 832 no. 1, (2017) 012010, arXiv:1703.09287 [nucl-ex] .

[16] N. Sharma, J. Mazer, M. Stuart, and C. Nattrass, "Background subtraction methods for precision measurements of di-hadron and jet-hadron correlations in heavy ion collisions," Phys. Rev. C93 no. 4, (2016) 044915.

[17] K. C. Zapp, “Geometrical aspects of jet quenching in JEWEL,” Phys.Lett. B735 (2014) 157-163.

[18] K. C. Zapp, “JEWEL 2.0.0: directions for use,” Eur. Phys. J. C74 no. 2, (2014) 2762, arXiv:1311.0048 [hep-ph].

[19] T. Sjostrand, S. Mrenna, and P. Z. Skands, "PYTHIA 6.4 Physics and Manual," JHEP 05 (2006) 026, arXiv:hep-ph/0603175 [hep-ph].

[20] P. Z. Skands, “Tuning Monte Carlo Generators: The Perugia Tunes,” Phys.Rev. D82 (2010) 074018, arXiv:1005.3457 [hep-ph].

[21] A. Majumder and J. Putschke, "Mass depletion: a new parameter for quantitative jet modification," Phys. Rev. C93 no. 5, (2016) 054909.

[22] ALICE Collaboration, S. Acharya et al., "First measurement of jet mass in $\mathrm{Pb}-\mathrm{Pb}$ and $\mathrm{p}-\mathrm{Pb}$ collisions at the LHC," arXiv:1702.00804 [nucl-ex] .

[23] ALICE Collaboration, L. Cunqueiro, "Jet shapes in pp and Pb-Pb collisions at ALICE," Nucl. Phys. A956 (2016) 593-596, arXiv:1512.07882 [nucl-ex]. 